\title{
Design of Monolithic RF Power Amplifier using Bulk BiCMOS Process
}

\author{
W.C. Chan, Philip K.T. Mok and Johnny K.O. Sin \\ Dept. of Electrical and Electronic Engineering \\ Hong Kong University of Science and Technology \\ Hong Kong SAR, China
}

\author{
W.T. $\mathrm{Ng}$ \\ Dept. of Electrical and Computer Engineering \\ University of Toronto, Toronto \\ Ontario, Canada
}

\begin{abstract}
A low-voltage monolithic 900MHz power amplifier has been fabricated in a commercial $0.8 \mu \mathrm{m}$ bulk BiCMOS process with an integrated output tuned circuit. The tuned circuit is implemented by a monolithic inductor of $2.6 \mathrm{nH}$ with $54 \mu \mathrm{m}$ metal width. The output power of the amplifier is $14 \mathrm{dBm}$.
\end{abstract}

\section{INTRODUCTION}

Radio frequency integrated circuit (RFIC) recently has aroused a lot of interest. The emphasis is currently placed on the development of low cost, low power and high performance communication ICs [1] to minimize the cost, weight and battery size of the handset.

For RF circuit design, Gallium Arsenide (GaAs) technology undoubtedly provides the best performance due to its low noise figure, high electron mobility, high gain and high output power [1]. However, the cost of using GaAs is extremely high and GaAs process is not compatible with the existing low cost IF CMOS circuit. As a result, most of the researchers in circuit design recently concentrate a lot of efforts in using CMOS technology to integrate the whole transceiver into one chip-set [2].

However, some RF CMOS building blocks still have unsatisfactory result compared with the other silicon technology - BJT. For example, frequency synthesizer exhibits larger phase noise in CMOS than BJT [1] [3]. Also, a BJT transistor has larger transconductance than a CMOS transistor under the same current. Therefore, BiCMOS process is chosen for the implementation of RFIC for the sake of good performance and higher integration. The RF power amplifier is implemented by the BJT while the other transceiver circuits are implemented by CMOS.

Monolithic inductor has been known to be a main obstacle for fully integrated RFIC. Up to now, most of power amplifier designs make use of the monolithic inductor in driver stage but rarely use it in output stage. This paper concentrates on the design of RF monolithic power amplifier using monolithic inductor to implement the tuned cir-

This work was supported by a grant from the Research Grants Council of the Hong Kong Special Administrative Region, China. (Project No. HKUST 789/96E) cuit in the final output stage by an existing bulk BiCMOS technology for low voltage and low power application.

\section{MONOLITHIC INDUCTOR}

Monolithic inductor has been widely used for RF IC implementation. However, there are some limitations such as high resistivity of the metal layer and substrate loss. Owing to the above limitations, the Q-factor of the monolithic inductor is only about 3 to 5 for CMOS and other similar processes.

To improve the Q-factor, several publications suggested to reduce the resistive loss by using thicker metal [4] and stacking of multi-metal layers [5] and to reduce the substrate loss by using thicker oxide, high resistivity silicon substrate [4] and removal of substrate beneath the spiral inductor by etching [6]. However, all of the above methods need to alter the process parameters, which is unavailable to circuit designers using existing silicon process.

Therefore, Q-enhancement circuit has been suggested to boost the Q-factor without changing process parameters. This method basically uses an active circuit to implement a negative resistance to compensate the power loss of the inductor [7]. However, as the Q-enhancement circuit also consumes power, the increase of total input power makes no effective gain on the overall efficiency and thus it is not suitable to be used in RF power amplifier design. Therefore, the monolithic inductor can only be optimized geometrically.

As power level of power amplifier is usually large, the metal width should be made large enough to meet the current requirement. However, it is suggested not to oversize the metal width as the parasitic capacitance will become large and it will greatly affect the inductive characteristic of the inductor. Also, the skin effect at high frequency limits the current capability of the metal line. Increasing the metal width will not have significant effect on the current handling capability.

The monolithic inductor for the RF power amplifier is optimized by a program called $A S I T I C$ [8]. The inductor coil is implemented by the second layer metal and the first layer metal is used to make the underpass of the coil. The design parameters and performance of monolithic induc- 
tor are summarized in Table I. The metal width of the inductor is $54 \mu \mathrm{m}$ and the inductance is about $2.6 \mathrm{nH}$. Figure 1 shows the plot of Q-factor against frequency. The maximum Q-factor is about 4.4. Also, it is found that the self-resonant frequency of the inductor is $3 \mathrm{GHz}$ and the inductor is suitable for $900 \mathrm{MHz}$ operation.

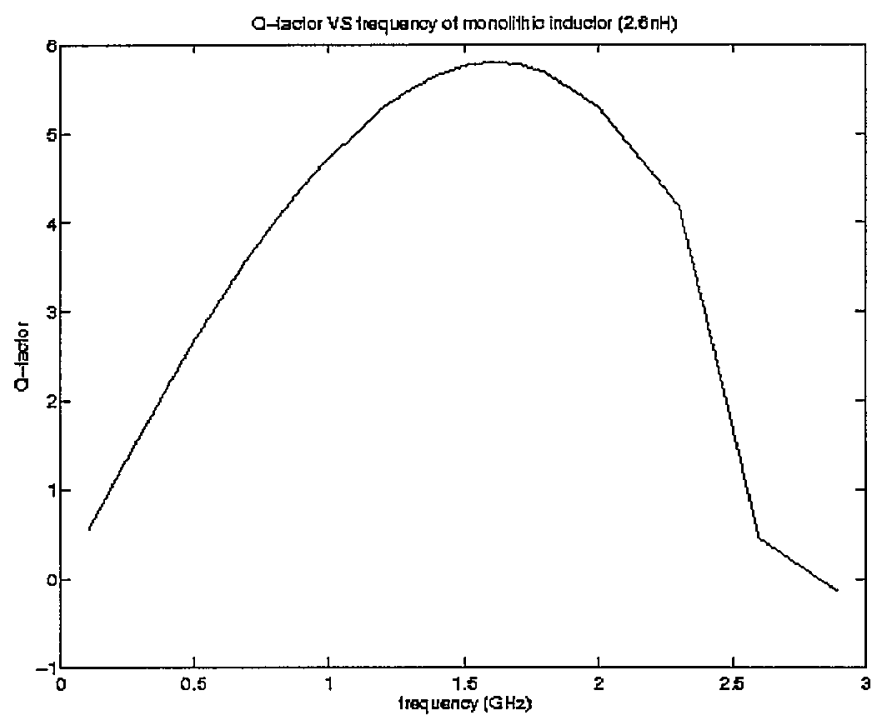

Fig. 1. Q-factor versus frequency of monolithic inductor.

TABLE I

Design parameters and performance of Monolithic Inductor used in output stage of Power Amplifier.

\begin{tabular}{|c|c|}
\hline Operating frequency & $900 \mathrm{MHz}$ \\
\hline Number of turns & 2.5 \\
\hline Outer length & $400 \mu \mathrm{m}$ \\
\hline Metal width & $54 \mu \mathrm{m}$ \\
\hline Metal separation & $3.8 \mu \mathrm{m}$ \\
\hline $\mathrm{L}$ & $2.623 \mathrm{nH}$ \\
\hline $\mathrm{Q}$ & 4.42 \\
\hline $\mathrm{R}$ & $3 \Omega$ \\
\hline Self-frequency & $3 \mathrm{GHz}$ \\
\hline Max. Q frequency & $1.65 \mathrm{GHz}$ \\
\hline
\end{tabular}

\section{RF POWER AMPLifier Design}

\section{A. Driver and Output Stage}

To design a RF power amplifier, the type of driver stage and output stage should be determined first. Therefore, different bipolar single output stage power amplifiers are compared. The single stage power amplifier was implemented by the BJT in AMS (Austria Miko Systeme Group, Austria) $0.8 \mu \mathrm{m}$ BiCMOS technology. It is a double-metal double-poly process with $12 \mathrm{GHz}$ high speed bipolar transistor. Figure 2 and Figure 3 compare the power gain and efficiency of Class AB, Class $C$ and Class E power amplifier. Each amplifier is designed to optimize their performance for $3 \mathrm{~V}$ supply voltage with $50 \Omega$ loading impedance. It is shown that Class $\mathrm{C}$ has the

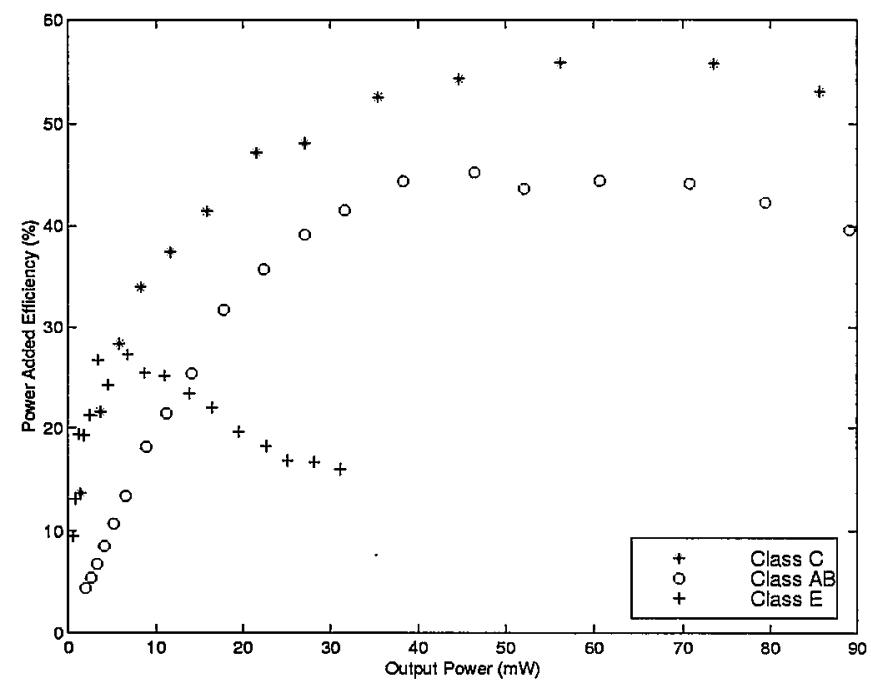

Fig. 2. PAE of different power amplifiers

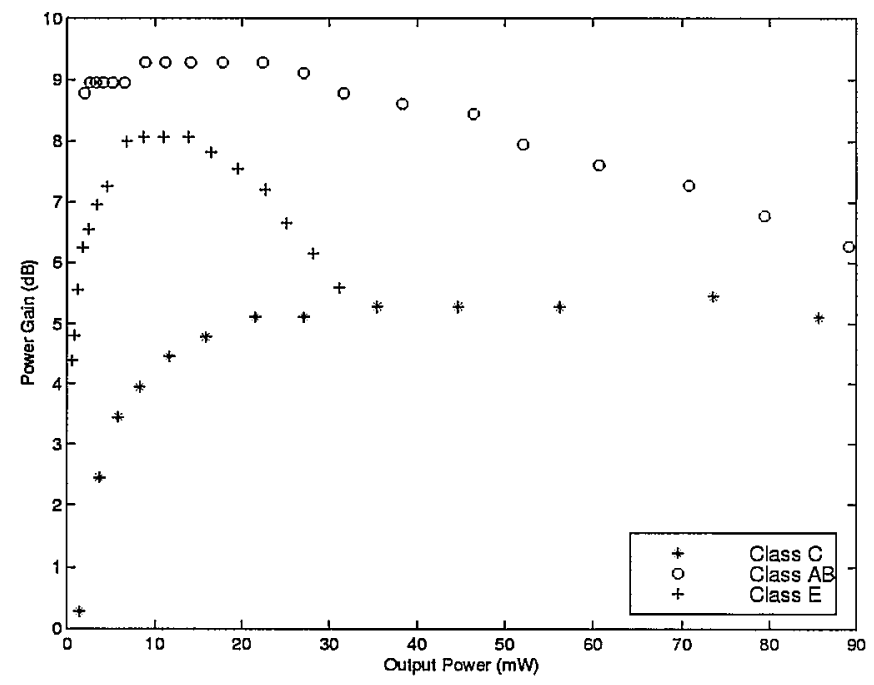

Fig. 3. Power gain of different power amplifiers

highest efficiency (55\%) and Class $\mathrm{AB}$ has the highest gain $(9.2 \mathrm{~dB})$. The performance of Class $\mathrm{E}$ is the poorest because of the deep-saturation of bipolar transistor leading to poor switching characteristic. Table II summarizes the measurement results of single stage power amplifiers. Based on the comparison results, Class AB is chosen to implement the driver stage because the power gain is high. Although the efficiency is low, the overall efficiency is not much affected as the driver stage will not dissipate large power compared with the output stage. Also, a cascode 
stage is added in the driver stage in order to block the parasitic capacitive feedback path to improve the stability and increase the signal gain. Class $\mathrm{C}$ is used to implement the output stage for high efficiency. Class $E$ is not preferred to implement output stage due to the poor switching characteristic of BJT and large switching stress. Moreover, the value of inductor used in Class $\mathrm{C}$ output tuned circuit $(2.6 \mathrm{nH})$ is significantly smaller than that in Class E $(39 \mathrm{nH})$ for same network Q-factor. Therefore, Class C output tuned circuit is easier to be integrated by present IC technology.

TABLE II

Summary of measurement results of power amplifiers with output stage only

\begin{tabular}{|c|c|c|c|}
\hline Class & C & AB & E \\
\hline Supply voltage (V) & 3 & 3 & 3 \\
\hline$P_{1 d B}(\mathrm{dBm})$ & 6.5 & 9 & 2 \\
\hline Max. power gain (dB) & 5.3 & 9.2 & 8 \\
\hline Max. drain efficiency (\%) & 68 & 48 & 28 \\
\hline Max. PAE (\%) & 55 & 45 & 27 \\
\hline
\end{tabular}

\section{B. Overall Design}

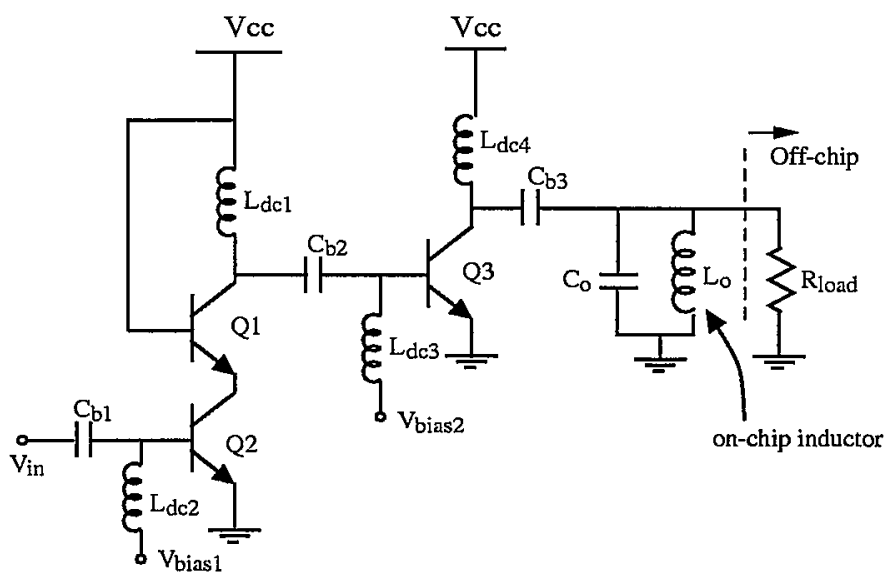

Fig. 4. Circuit of Monolithic Power Amplifier.

The overall circuit of monolithic power amplifier is shown in Figure 4. Q1 and Q2 form a Class AB driver stage with cascode configuration. Q3 is the Class $\mathrm{C}$ output stage with output tuned circuit $C_{o}$ and $L_{o}$. Due to low-power application, the impedance transformation matching network is not included to increase the power level. In fact, because of the low $\mathrm{Q}$-factor of the monolithic inductor, including the impedance matching network will dissipate more power and the overall efficiency of the amplifier is decreased. The output impedance level is

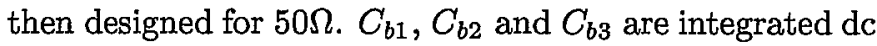
blocking capacitors implemented by poly-poly capacitors available in the process. $L_{o}$ is on-chip inductor but $L_{d c 1}$,
$L_{d c 2}, L_{d c 3}$ and $L_{d c 4}$ are off-chip inductors. The design parameters of the on-chip inductor have been discussed in the previous section.

\section{MEAsurement Results}

The monolithic power amplifier shown in Figure 4 was implemented by AMS $0.8 \mu \mathrm{m}$ BiCMOS process. Figure 5 shows the die photo of the monolithic power amplifier. The active area of the power amplifier is $0.96 \mathrm{~mm}^{2}$. Figure 6 to 8 show the measurement results of the amplifier. Under $3 \mathrm{~V}$ supply voltage operating at $900 \mathrm{MHz}$, the 1$\mathrm{dB}$ compression point is $9 \mathrm{dBm}$ as indicated in Figure 6. The maximum power gain and maximum PAE are $16 \mathrm{~dB}$ and $12 \%$, as shown in Figure 7 and 8 respectively. The low efficiency is mainly due to the losses in the interstage matching and the monolithic inductor. Table III shows the summary of performance of the monolithic power amplifier.

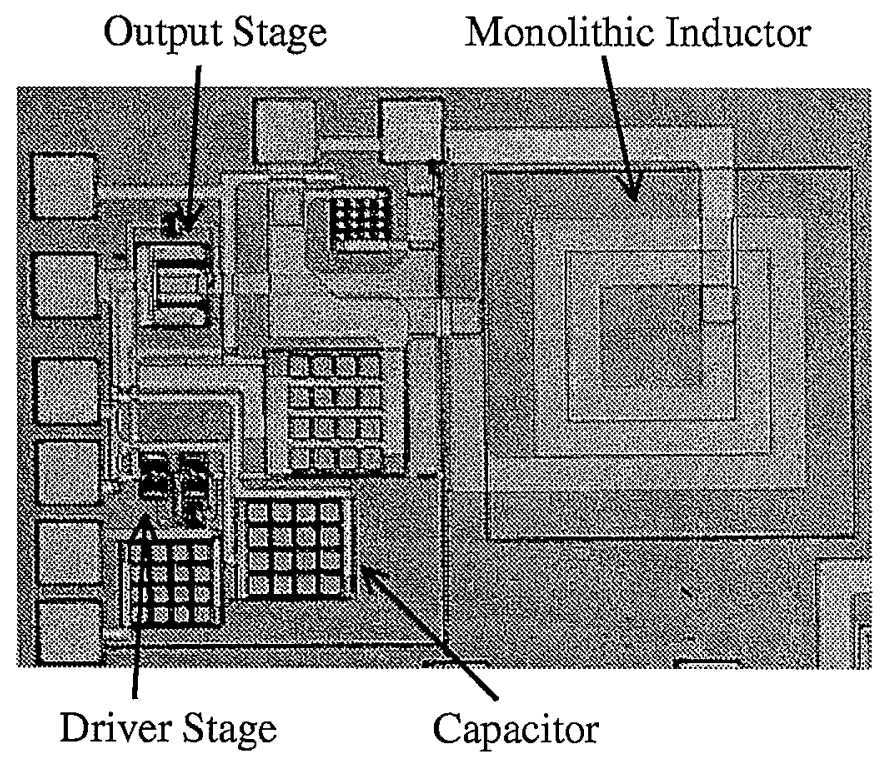

Fig. 5. Micrograph of the monolithic power amplifier

TABLE III

Summary of measurement results of monolithic power amplifier operating at $900 \mathrm{MHz}$

\begin{tabular}{|c||c|}
\hline Supply voltage (V) & 3 \\
\hline Technology & $0.8 \mu \mathrm{m} \mathrm{BiCMOS}$ \\
\hline Driver stage & Class AB \\
\hline Output stage & Class C \\
\hline$P_{1 d B}$ & $9 \mathrm{dBm}$ \\
\hline Max. Output Power & $17 \mathrm{dBm}$ \\
\hline Max. power gain & $16 \mathrm{~dB}$ \\
\hline Max. drain efficiency & $12 \%$ \\
\hline Max. PAE & $11.7 \%$ \\
\hline Active area & $0.96 \mathrm{~mm}^{2}$ \\
\hline
\end{tabular}




\section{Conclusion}

This work demonstrates that a low-voltage RF power amplifier with fully integrated output tuned circuit can be implemented by a standard $0.8 \mu \mathrm{m}$ bulk BiCMOS process with $12 \mathrm{GHz}$ high speed bipolar transistor and it is suitable for indoor cordless phone application. The performance of the power amplifier can be enhanced if a process with multi-metal layers and high resistivity substrate is used. In this way, the Q-factor of monolithic inductor can be higher and the efficiency of power amplifier can be further increased.

\section{ACKNOWLEDGMENT}

I would like to thank the members in WCL, IPEL and VLSI Laboratory for their assistance in CAD tools, onwafer measurement and other technical supports. Also, I am indebted to Mr. Ali M. Niknejad for providing the program of $A S I T I C$ on inductor simulation.

\section{REFERENCES}

[1] Lawrence E. Larson. Integrated circuit technology options for RFIC's - present status and future directions. IEEE Journal of Solid-State Circuits, Vol. 33, March 1999.

[2] Ahmadreza Rofougaran, Glenn Chang, Jacob J. Rael, James Y. C. Chang, Maryam Rofougaran, Paul J. Chang, Masoud Djafari, M. K. Ku, Edward W. Roth, Asad A. Abidi, and Henry Samueli. A single-chip $900-\mathrm{MHz}$ spread-spectrum wireless transceiver in 1- $\mu \mathrm{m}$ CMOS. IEEE Journal of Solid-State Circuits, Vol. 33, April 1998.

[3] M. Bonford. Selection of frequency dividers for microwave PLL applications. Microwave $J$, Nov 1990.

[4] K. B. Ashby, I. A. Koullias, W. C. Finley, J. J. Bastek, and S. Moinian. High $Q$ inductors for wireless applications in a complementary silicon bipolar process. IEEE Journal of SolidState Circuits, Vol. 31, January 1996.

[5] J. N. Burghartz, M. Souyer, and K. A. Jenkins. Microwave inductors and capacitors in standard multilevel interconnect silicon technology. IEEE Trans. Microwave Theory Tech., Vol. 44, January 1996.

[6] J. Y.-C. Chang, A. A. Abidi, and M. Gaitan. Large suspended inductors on silicon and their use in a 2- $\mu \mathrm{m}$ CMOS RF amplifier. IEEE Journal of Solid-State Circuits, Vol. 33, May 1998.

[7] Chung-Yu Wu and Shuo-Yuan Hsiao. The design of a 3-V 900$\mathrm{MHz}$ CMOS bandpass amplifier. IEEE Journal of Solid-State Circuits, Vol. 32, February 1997.

[8] Ali M. Niknejad and Robert G. Meyer. Analysis and optimization of monolithic inductors and transformers for RF ICs. IEEE Custom Integrated Circuits Conference, 1997.

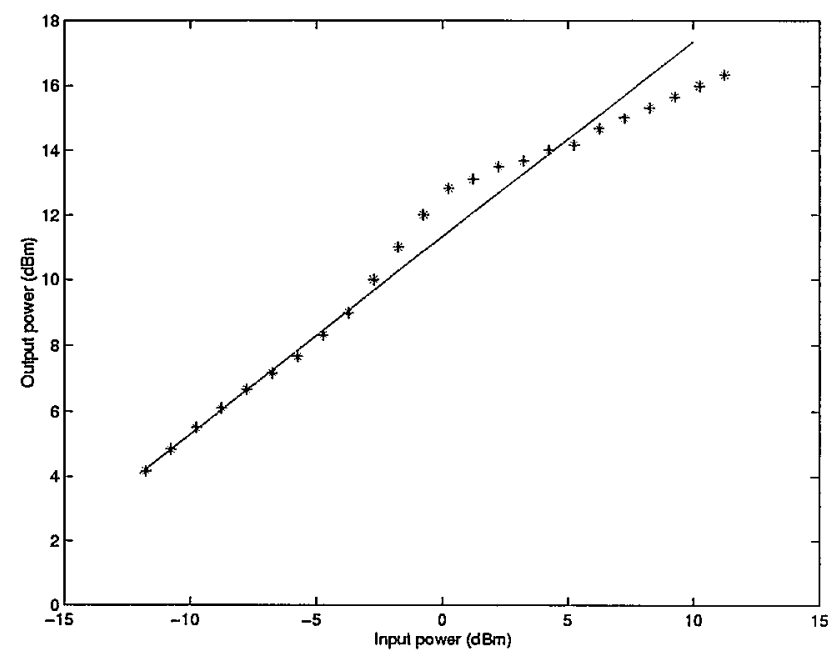

Fig. 6. Output power versus input power of monolithic power amplifier

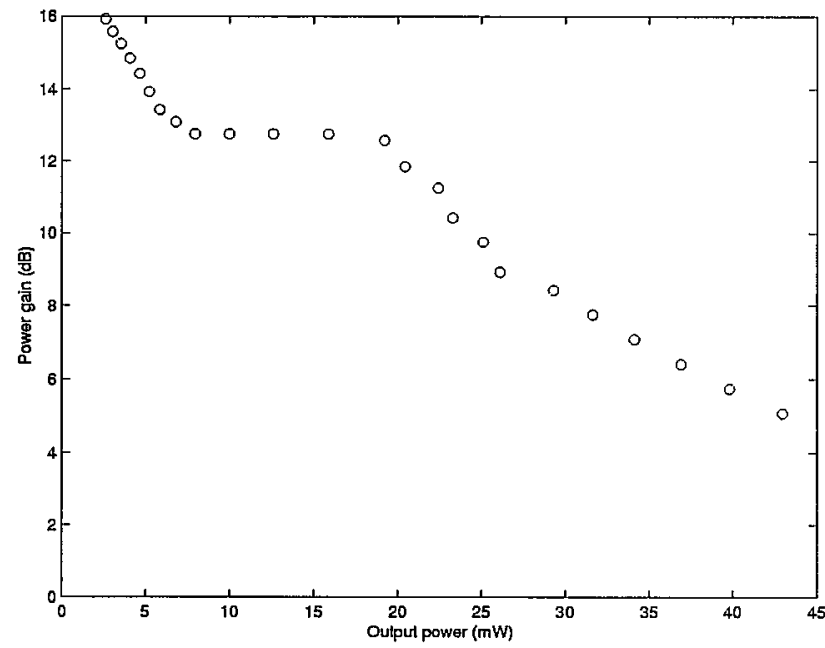

Fig. 7. Power gain of monolithic power amplifier

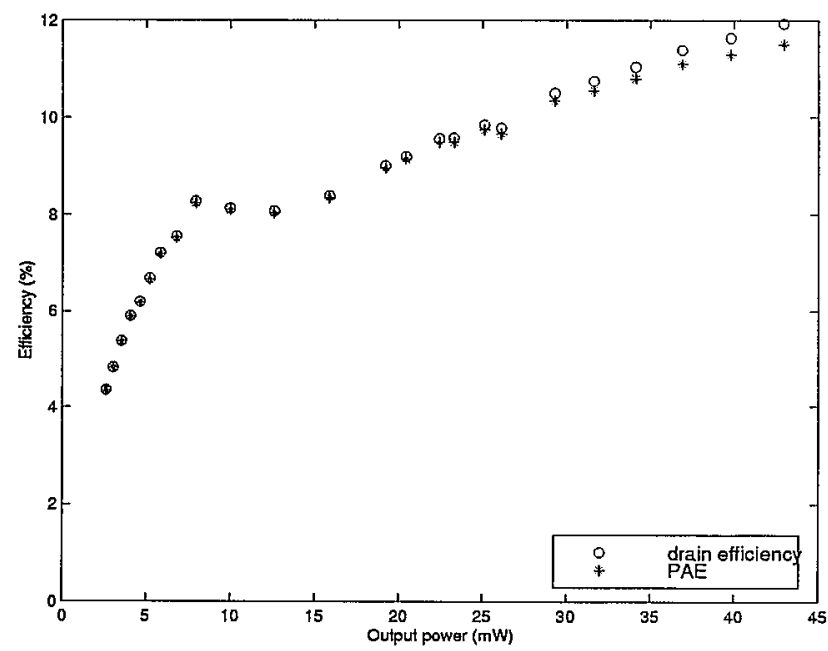

Fig. 8. Efficiency of monolithic power amplifier 